\title{
Line shape analysis of electron-hole plasma electroluminescence in fully strained SiGe epitaxial layers
}

\author{
T. Stoica ${ }^{\mathrm{a})}$ and L. Vescan \\ Institut für Schichten und Grenzflächen (ISG), Forschungszentrum Jülich GmbH, D-52425 Jülich, Germany
}

(Received 21 April 2003; accepted 16 July 2003)

\begin{abstract}
The electroluminescence of $p-i-n$ diodes with fully strained $\mathrm{Si}_{0.80} \mathrm{Ge}_{0.20} / \mathrm{Si}(001)$ is dominated by radiative recombination in an electron-hole plasma. The recombination mechanisms and the band gap renormalization have been studied experimentally and by modeling. In order to minimize the influence of the $\mathrm{SiGe} / \mathrm{Si}$ interface regions and thus to study the intrinsic behavior of strained $\mathrm{SiGe}$, electroluminescence diodes with thick layers of $\mathrm{SiGe}$ in a metastable strain state have been investigated. To explain the electroluminescence spectra, the band filling model for an electron-hole plasma system and different broadening procedures have been investigated. This line shape analysis allowed the determination of the dependence of the renormalized band gap on carrier density and comparison with theoretical predictions was done. The low-energy tails of the electroluminescence spectra correspond to a broadening of the initial electronic states of the recombination process in electron-hole plasma system. The experimental data obtained from the line shape analysis of the electroluminescence spectra are in good agreement with previous results on spectral photocurrent and quantum efficiency measurements. (c) 2003 American Institute of Physics.
\end{abstract}

[DOI: $10.1063 / 1.1606513]$

\section{INTRODUCTION}

The use of germanium in thick $\mathrm{SiGe}$ alloys, in $\mathrm{SiGe} / \mathrm{Si}$ quantum wells, and in self-assembled quantum dots is of a great interest for integrated optoelectronics. ${ }^{1}$ However, light emitters with reasonable quantum efficiency based on SiGe were not yet realized, the main obstacle being the indirect band gap character of this material. In spite of this character, no-phonon (NP) beside phonon-assisted transitions are observed in photo- and electroluminescence (EL) of SiGe single- and multiple-quantum-well layers at low temperatures. ${ }^{2-4}$ The no-phonon transitions can appear due to fluctuations of alloying, doping, and strain, as well as by quantum confinement. At room temperature, the efficiency decreases by thermal activation of the injected carriers into the surrounding $\mathrm{Si}$. By improving the diode structure, EL near the optical communication wavelength of $1.3 \mu \mathrm{m}$ can be obtained with SiGe diodes with an internal quantum efficiency $(\mathrm{QE})$ of $\mathrm{QE}_{\mathrm{int}}=0.1 \%,{ }^{5}$ while for nominally pure $\mathrm{Ge}$ islands, the diodes emit near $\lambda=1.55 \mu \mathrm{m}$ with $\mathrm{QE}_{\text {int }}$ $=0.001 \% .^{6}$ The plastic relaxation of thick SiGe layers can be avoided and a metastable strain state well above the critical thickness can be obtained by selective epitaxial growth on small area. ${ }^{7}$

The optoelectronic properties and the QE of our SiGe diodes have been previously investigated. ${ }^{5,8-10}$ To understand the EL spectra for the further improvement of the QE, the properties of the high-density electron-hole system in thick fully strained $\mathrm{Si}_{0.80} \mathrm{Ge}_{0.20}$ layers have been analyzed in the present article in more detail.

a) Author to whom correspondence should be addressed; electronic mail: t.stoica@fz-juelich.de
At a high density of the injected carriers, the excitonic system is transformed into an electron-hole plasma (EHP) by the metal-insulator Mott transition. The exciton binding energy is reduced to zero at the Mott transition density due to the screening of the electron-hole Coulomb interaction in the presence of many free carriers. The Mott critical density at temperature $T$ can be estimated using the formula $n_{\text {Mott }}$ $=1.4 \epsilon / 8 \pi e^{2} a_{B}^{2} K T$ where $a_{B}$ is the Bohr radius of the exciton, and $\epsilon$ the static dielectric constant. For Si, the estimated $n_{\text {Mott }}$ value for $80 \mathrm{~K}$ is about $10^{17} \mathrm{~cm}^{-3}$. The aforementioned formula deduced in a Debye-Hückel model is a good hightemperature approximation experimentally verified for $\mathrm{Si}$ and Ge above the liquid-gas critical temperature. ${ }^{11,12}$ For a lower temperature, an approximation based on the ThomasFermi screening length can be written (see, for example, Ref. 13). By a more complex theory based on random-phase approximation, an extrapolation at $0 \mathrm{~K} n_{\text {Mott }}=3 \times 10^{16} \mathrm{~cm}^{-3}$ can be estimated for $\mathrm{Si}^{11}$

Below a critical temperature, the electron-hole system in $\mathrm{Si}$ and $\mathrm{Ge}$ can be in a gas or liquid phase of excitons. Depending on the concentration and temperature, the condensation of excitons into electron-hole droplets (EHDs) takes place. ${ }^{14-18}$ The carrier density within the droplets decreases by increasing the temperature, but remains much higher than the Mott critical value. For $\mathrm{Si}$, the pair density within droplets is $3.5 \times 10^{18} \mathrm{~cm}^{-3}$ at $T=5 \mathrm{~K}$ and decreases to $1.2 \times 10^{18} \mathrm{~cm}^{-3}$ at the critical temperature of $23 \mathrm{~K}^{15,16}$ For a uniform EHP, the plasma density and the shape of the luminescence spectra change with carrier injection level, while for EHD the carrier density and the spectrum shape remain almost constant with the injection intensity, only the droplet volume and the light emission intensity change. 
TABLE I. Data of $\mathrm{Si}_{1-x} \mathrm{Ge}_{x} / \mathrm{Si}$ LED structures (the samples were ordered after the SiGe thickness; the buffer, and SiGe layer have a $n$-type doping).

\begin{tabular}{|c|c|c|c|c|c|c|c|}
\hline $\begin{array}{c}\text { Sample } \\
\text { No. }\end{array}$ & $\begin{array}{c}\text { Buffer } \\
\text { thickness } \\
(\mathrm{nm})\end{array}$ & $\begin{array}{l}\text { Buffer } \\
\text { doping } \\
\left(\mathrm{cm}^{-3}\right)\end{array}$ & $\begin{array}{c}\mathrm{Ge} \\
\text { content } \\
x\end{array}$ & $\begin{array}{c}n \text {-SiGe } \\
\text { thickness } \\
(\mathrm{nm})\end{array}$ & $\begin{array}{l}n-\mathrm{SiGe} \\
\text { doping } \\
\left(\mathrm{cm}^{-3}\right)\end{array}$ & $\begin{array}{c}\text { Spacer } \\
\text { thickness } \\
(\mathrm{nm})\end{array}$ & $\begin{array}{c}\text { Top } \\
\text { contact }\end{array}$ \\
\hline 1597 & 230 & $\sim 2 \times 10^{17}$ & 0 & 0 & $\ldots$ & $\ldots$ & $\mathrm{ZnO}$ \\
\hline 696 & 350 & $\sim 5 \times 10^{16}$ & 0.20 & 60 & $\sim 1 \times 10^{16}$ & 60 & $\mathrm{Al} / \mathrm{Au}$ \\
\hline 1296 & 260 & $8 \times 10^{17}$ & 0.21 & 260 & $1 \times 10^{18}$ & 30 & $\mathrm{Al} / \mathrm{Au}$ \\
\hline 1687 & 580 & $8 \times 10^{17}$ & 0.21 & 445 & $1 \times 10^{18}$ & 24 & $\mathrm{ZnO}$ \\
\hline
\end{tabular}

Due to the relative long lifetime of the carriers in highquality SiGe layers, the carrier density is higher than $n_{\text {Mott }}$ at a moderate injection level. For quantum well structures under normal injection conditions, the carrier density exceeds Mott density more easily, thus, the collective phenomena in the EHP have to be taken into account. 19,20

In this work, the uniform EHP in SiGe is studied by an investigation of EL in SiGe fully strained epitaxial layers. The renormalized band gap of the EHP, dependent upon carrier concentration, was obtained by the line shape analysis of EL spectra and was compared with theoretical predictions. The low-energy tail modeling gives information about the energy uncertainty of the unielectron states involved in radiative recombination in the EHP system.

\section{EXPERIMENTAL DETAILS}

Diodes in the form of mesas (size 20-200 $\mu \mathrm{m}$ ) were selectively grown by low-pressure chemical vapor deposition at $700{ }^{\circ} \mathrm{C}$ with the layer sequence $n^{+} n p^{+}$: (1) $n^{+}-\mathrm{Si}$ substrate, (2) $n^{+}$-Si buffer, (3) $n$-Si buffer, (4) $n$ - $\mathrm{Si}_{1-x} \mathrm{Ge}_{x}(x$ $=0.20-0.28$, thickness: $70-450 \mathrm{~nm}),(5) n-\mathrm{Si}_{1-x} \mathrm{Ge}_{x}(x$ $=0.02 ; 20-60 \mathrm{~nm})$, and (6) boron-doped $\mathrm{Si}_{1-x} \mathrm{Ge}_{x}(x$ $=0.02$, thickness $\sim 100 \mathrm{~nm}$ ). The ohmic front and bottom contacts were evaporated $\mathrm{Al} / \mathrm{Au}$ (or $\mathrm{ZnO}$ ) and $\mathrm{AuSb}$, respectively. Other deposition and diode fabrication details are given in Refs. 9, 21, and 22. The samples investigated are described in Table I. The EL spectra were measured using a Fourier transform spectrometer (BIO RAD FTS40). The doping profile was obtained from secondary ion mass spectroscopy (SIMS) measurements and the thickness of SiGe and Ge concentration from Rutherford backscattering spectroscopy (RBS) data. SIMS depth profiles were performed using a quadrupole instrument (Atomika 4000). RBS data were obtained using a 1.7 MV Tandetron accelerator.

\section{EXPERIMENTAL DATA}

\section{A. Electroluminescence spectra}

EL spectra of fully strained diodes have been measured in the temperature range of $16-300 \mathrm{~K}$. At a given temperature, the shape and peak energies of the spectra depend on the injection level. Therefore, in order to compare the spectra of different diodes, similar injection conditions must exist. Spectra of different samples are shown in Fig. 1 for a current density of $\sim 60 \mathrm{~A} \mathrm{~cm}^{-2}$ and a temperature range of $50-80 \mathrm{~K}$, in comparison with the EL of pure Si diodes. The SiGe diodes have similar behavior with well resolved NP and transversal optical (TO) phonon-assisted transitions peaks at nearly the same energetic positions for all of the samples: $E_{\mathrm{NP}}=1005-1007 \mathrm{meV}$ and $E_{\mathrm{TO}}=954-955 \mathrm{meV}$. The EL of pure Si diodes has a broad peak at $1078 \mathrm{meV}$ at $50 \mathrm{~K}$ and the intensity remains almost constant up to room temperature. At higher temperatures, the broad NP and TO peaks of SiGe are partially superposed and an additional contribution from $\mathrm{Si}$ can be observed. The Si contribution observed for SiGe diodes increases with current injection and temperature and decreases with SiGe thickness $d_{\mathrm{SiGe}} \cdot{ }^{5}$

\section{Band filling effect}

The high injection under EHP conditions corresponds to the band filling effect, i.e., the quasi-Fermi level lies in the band, at least for one of the bands (in strained SiGe, the valence band has a higher quasi-Fermi level relative to the band-see the discussion in Sec. IV C). The band filling can be observed experimentally by the effects on EL spectra (peak position $E_{\mathrm{NP}}$ and peak width-Figs. 2 and 3), as well as by the effect on the temperature dependence (activation energy). ${ }^{5}$ EL integral intensity decreases at higher temperatures due to the thermal activation of holes from the SiGe well into Si regions. The activation energy decreases by increasing the injection current due to the shift of the quasiFermi level relative to the bottom of the band.

In Figs. 2(a) and 2(b), the spectra are shown in logarithmic scale for different injection currents and temperatures. The NP and different phonon replicas of the SiGe EL can be observed, as well the low and high-energy tails. For energies

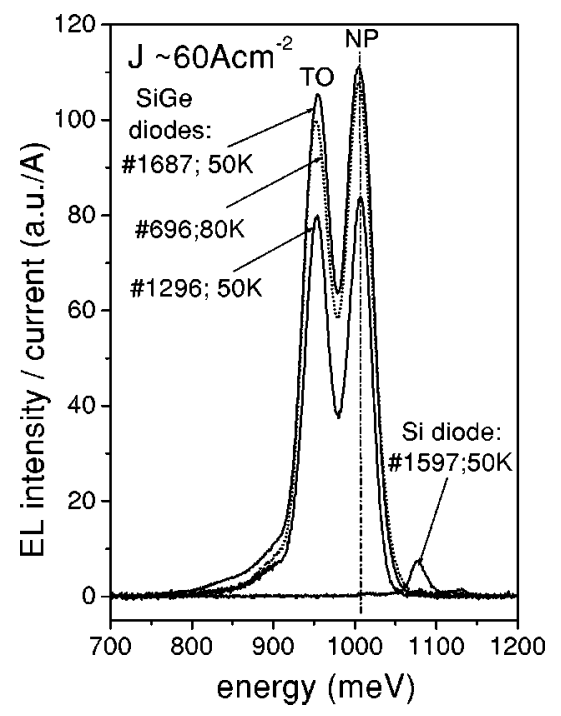

FIG. 1. Comparison of low temperature EL spectra of light-emitting diodes with $\mathrm{Si}$ (No. 1597) and $\mathrm{SiGe}\left[d_{\mathrm{SiGe}}=60,260\right.$, and $445 \mathrm{~nm}$ of samples Nos. 696 (see Ref. 23), 1296, and 1687]. 


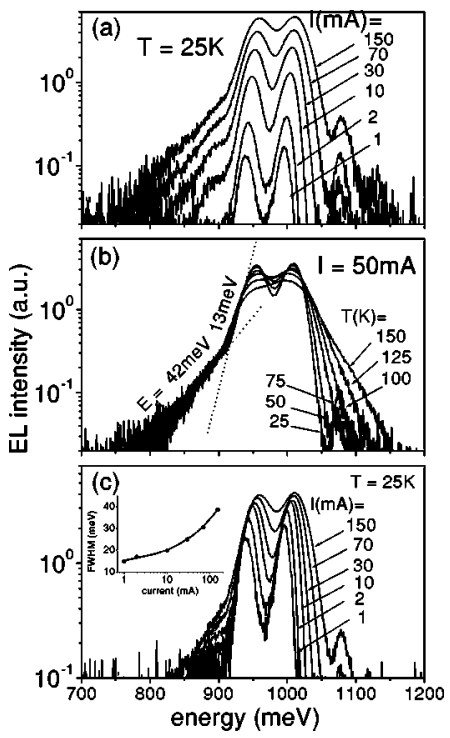

FIG. 2. Strained-SiGe EL spectra on logarithmic scale: (a) Spectra for different current values, at $25 \mathrm{~K}$; (b) spectra for different temperatures, at 50 $\mathrm{mA}$; (c) spectra of figure (a) but normalized to obtain a tangent-curves family (inset: The current dependence of the peak width—full width at half maximum).

above $1070 \mathrm{meV}$, the $\mathrm{Si}$ emission is also observed at higher currents or temperatures. Figure 2(c) shows the curves of Fig. 2(a) but shifted on the logarithmic scale to obtain tangent of curves on the low-energy side of the maxima. The width of the maxima clearly increases with current [see, also, the inset of the Fig. 2(c)], therefore, the band filling model is suggested to be suitable for the shape analysis.

However, while the peak width is always increasing with the current due to the band filling effect, the shift of the peak position to a higher energy can be compensated for by the decrease of the renormalized gap [Fig. 3(a); see also Sec. IV C].

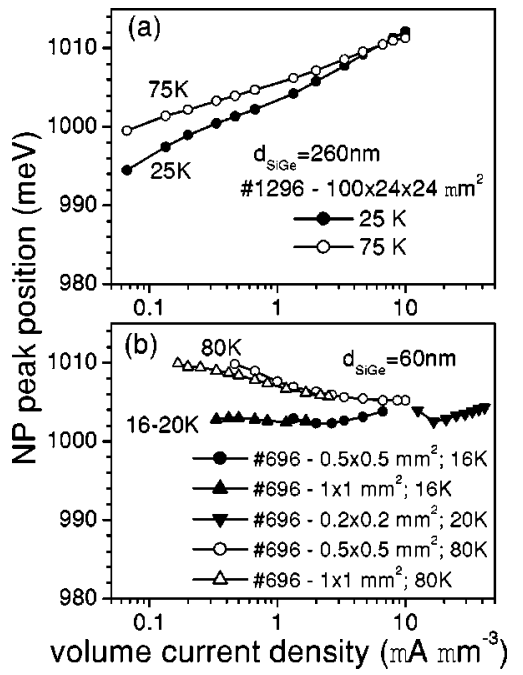

FIG. 3. NP peak position $E_{\mathrm{NP}}$ versus volume current density $J_{\text {vol }}$ for samples with (a) and without (b) doping of the bottom part of the buffer layer. Full symbols are for lower temperatures $16-25 \mathrm{~K}$ and open symbols for higher temperatures 75-80 K. Sample No. 1296 consists of an array of 100 diodes of an area $24 \times 24 \mu \mathrm{m}^{2}$. Sample No. 696 is single diodes.

\section{Influence of doping}

At a low temperature, a doped buffer is observed to have only a slight influence on the spectra. This is due to the fact that recombination takes place only in $\mathrm{SiGe}$, i.e., far away from the buffer/substrate interface. Similar spectra can be seen in Fig. 1 for samples with or without doping of the bottom part of the buffer layer. However, there are qualitative differences between the two diode types regarding the current injection dependency of the NP and TO peak positions. For a better comparison, the volume current density dependence was used ( $J_{\text {vol }}$-volume current density defined as the ratio of injection current to $\mathrm{SiGe}$ volume). Figures 3(a) and 3(b) show, at low temperatures, the dependencies $E_{\mathrm{NP}}\left(J_{\mathrm{vol}}\right)$ for samples No. 1296 (with a doped buffer) and for No. 696 (without a doped buffer), respectively (see Table I). Sample No. 1296 shows a monotonic increase of $E_{\mathrm{NP}}$ at both temperatures of $25 \mathrm{~K}$ and $75 \mathrm{~K}$. For sample No. 696, $E_{\mathrm{NP}}$ is nearly constant at $16-20 \mathrm{~K}$ and decreases with $J_{\text {vol }}$ at $80 \mathrm{~K}$. The qualitative difference between the behavior of the peak position $E_{\mathrm{NP}}$ with current can be explained by the different residual doping of the SiGe layer. We will see in Sec. IV that the interpretation of the line shape and peak position is a matter of the analysis of the radiative recombination in an EHP system. Here, many factors such as band structure, injection level, temperature, and doping were taken into account. The narrowing of the band gap by collective interaction (band-gap renormalization) compensates for the blueshift of $E_{\mathrm{NP}}$ due to the band filling effect. The gap narrowing given by doping must be added to the gap narrowing of the EHP effect. The residual doping of $\sim 10^{18} \mathrm{~cm}^{-3}$ in SiGe of sample No. 1296 results in a significant band-gap narrowing and the additional EHP may have less of an influence on the band-gap renormalization. In this case, the band filling effect might be higher than the EHP-induced band-gap narrowing and the peak position makes a blueshift with injection.

In the next sections, the interpretation of the EL spectra will be given on the basis of the EHP model.

\section{LINE SHAPE ANALYSIS}

\section{A. Electron-hole plasma in SiGe strained layers}

The critical temperature for the liquid-gas transition of the excitonic system in $\mathrm{Si}$ decreases under an external applied stress. The band structure is modified under stress, i.e., the number of subbands and equivalent minima which are essentially occupied by the injected carriers changes by changing the stress type. Strained $\mathrm{Si}_{1-x} \mathrm{Ge}_{x} / \mathrm{Si}(001)$ has four equivalent minima in the conduction band. For the valence band, the heavy hole band has the highest energy. ${ }^{24}$ The same type of band structure is for $\mathrm{Si}$ under uniaxial (110) compressive or (001) tensile stress, i.e., four equivalent minima in the conduction band and one maximum in the valence band. For Si under uniaxial (110) compressive stress, a reduction of the critical temperature from $23 \mathrm{~K}$ to $16 \mathrm{~K}$ was found. ${ }^{16}$ The critical temperature for liquid-gas transition in Ge is even lower, about $7 \mathrm{~K}^{17}$ Therefore, we can expect the critical temperature in SiGe strained layers to be reduced by 
strain and also by the addition of Ge. Thus, our experimental data in the range of $20-80 \mathrm{~K}$ should correspond to an uniform spatial distribution of the EHP.

In the EHP system, the collective interaction makes three contributions to the electron-hole energy of an uniparticle description: exchange, correlation and Hartree energies. ${ }^{25}$ In three-dimensional (3D) systems, the Hartree energies of holes and electrons compensate for each other due to the local neutrality, in contrast to low-dimensional systems.

There are extensive theoretical studies devoted to the calculation of the exchange and correlation energies for a 3D EHP. Vashishta and Kalia ${ }^{26}$ have published an "universal" formula for the sum of the exchange and correlation energy $E_{\text {ex }}$ :

$$
E_{\mathrm{ex}}=\frac{a+b r_{s}}{c+d r_{s}+r_{s}^{2}} \mathrm{Ry} *
$$

where $r_{s}=\left[3 /\left(4 \pi a_{B}^{3} n\right)\right]^{1 / 3}$ is a normalized interparticle distance with density $n$ of electron-hole pairs and Ry* is the Rydberg energy. The material-independent constants are $a$ $=-4.8316, b=-5.0879, c=0.0152$, and $d=3.0426$. With the equation for the exchange-correlation energy, the renormalized bandgap can be written:

$$
E_{\mathrm{g}}^{*}=E_{g}+\frac{\partial}{\partial n}\left(n E_{\mathrm{ex}}\right),
$$

where $E_{g}$ is the intrinsic gap. The Vashishta and Kalia formula was shown to be applicable to a number of nonpolar semiconductors. ${ }^{17,18}$

\section{B. Electron-hole plasma line shape model}

Besides the NP peak, the highest contribution of the phonon-assisted emission is from the TO replica. In the uniparticle description of the EHP interacting multiparticle system, the electrons and holes are distributed on electronic states in conduction and valence bands. The thermal relaxation of the carriers within the bands is a fast phenomenon and the energetic distribution of carriers can be described by the Fermi function with quasi-Fermi levels $\epsilon_{\mathrm{F}}^{\mathrm{e}}$ and $\epsilon_{\mathrm{F}}^{\mathrm{h}}$ for electrons and holes, respectively. As can be seen by numerical simulation of the carrier injection in $\mathrm{SiGe}$ diodes $^{9}$ for high injection levels and for $\mathrm{d}_{\mathrm{SiGe}}=70-400 \mathrm{~nm}$, the carriers have a quasi-uniform distribution and the quasi-neutrality is satisfied in the SiGe region. Under these conditions, the radiative band-to-band recombination at a given energy $h \nu$ of the emitted photon is an integral over the carrier density in the valence and conduction bands. The theoretical approach of the EL spectra of EHP is given in Appendix A.

The total emission is given by superposition of the NP spectrum and all-phonon replicas with different ratios phonon/NP of the light emission $r_{\mathrm{i}}$ [Eq. (A3)]. The computed spectra depend on a renormalized energy gap, quasi-Fermi levels, local temperature, and band distribution densities.

For the nondegenerate case, the quasi-Fermi levels are in the energy gap and the occupation probability of the band states is well described by the Boltzmann distribution. This is the case of moderate injection level and higher temperature, when the shape of the emission spectra depends only on temperature and not on the injection level.

At a high injection level and low temperature, the EHP corresponds to the degenerate condition, at least for one band. The shape of the emission peak depends on the band filling (carrier injection level) and temperature. In this case, by fitting the computed spectral shape [Eqs. (A1)-(A4)] to the experimental curves, the values of the plasma density, the local temperature, and renormalized band gap can be evaluated.

The high-energy tail of the emission spectra can be well described with Eq. (A1) by assuming thermal activation of the carriers above the quasi-Fermi levels. However, the experimentally observed tail at the low-energy side [Fig. 2] cannot be explained by this equation. Equation (A1) is based on the assumption of parabolic bands $D_{h}(\epsilon) \sim \epsilon^{1 / 2}$ without broadening of the electronic levels.

The low-energy tail of the emission spectra can be reproduced by replacing the $\delta$ distribution function in Eq. (A1) with a broad function such as Gauss or Lorentz functions, or a distribution function based on $\cosh ^{-1}(x) .{ }^{27}$ The Gauss function is appropriate for statistical fluctuations (alloy, doping, and/or thermal fluctuations). The Lorentz function is used to describe the uncertainty of energy levels due to a short lifetime. The $\cosh ^{-1}(x)$ has no physical meaning but can describe the experimentally observed exponential tails. An exponential tail of the band state distribution was also used to describe the disorder effect and to explain the low-energy tails of the emission spectra. ${ }^{28}$

Phenomena, like partial Auger recombination or plasmon interaction, may lead to low-energy tails of the spectra. ${ }^{29,30}$ Landsberg pointed out that the final state of a recombination process has a strong lifetime broadening in a degenerate electron gas. ${ }^{31}$ The carrier, which recombines radiatively, leaves a hole below the Fermi level and this excited state of the electron and hole ensemble has a short lifetime. It results in a broadening of the energy levels of the carriers involved in the recombination process. The broadening function is Lorentz type which gives the probability of having an effective photon energy different from the difference of the energies of the uniparticle initial states of the recombining the electron and hole. In this case, the electron and hole carrier distribution functions $\rho_{e, h}(\epsilon)$ must be replaced by the convolutions of the carrier distribution with the Lorentz function [Eq. (A5)] and the EL spectra are computed with Eq. (A6). The broadening parameter $\Gamma_{e, h}(\epsilon)$ of the Landsberg theory may be described by the simplified Eq. (A8).

A good agreement of the Landsberg broadening theory with experimental data was reported by Martin and Störmer ${ }^{14}$ and by $\mathrm{Schmid}^{15}$ for EHP in Ge and $\mathrm{Si}$, respectively. In comparison to the no-broadening approach, some changes of the plasma parameters were obtained by considering the Landsberg broadening. However, disagreement of the Landsberg broadening with the experiments was reported, too. ${ }^{17,18,32}$

Another broadening of the spectra can occur due to the uncertainty of the initial electron and hole states of the uni- 
particle approximation in Eqs. (A1) and (A6). The lifetime of the uniparticle states may be very short due to the multiparticle interaction in the dense EHP. In the case of the initial state broadening, the Fermi function is taken out of the convolution integral in Eq. (A5). For a real case, energy broadening for both initial and final states has to be considered.

\section{Results of line shape analysis of electroluminescence spectra}

In general, it is accepted that taking the low-energy tails into account only slightly affects the value of the plasma parameters which can be found by fitting to the experimental data, in comparison with a fit without tails. The differences increase with injection level and/or temperature when the NP maximum and phonon replica are not well resolved.

Figure 2(a) shows curves at $25 \mathrm{~K}$ for various current values. The tails of the spectral maxima are put into evidence in logarithmic scale. The main contribution to the spectrum is given by the NP and TO maxima of the SiGe layer. On the high-energy side, the decreasing slope depends on temperature in agreement with the thermal activation of the carriers to higher-energetic levels. At low injection currents, a shoulder due to the $\mathrm{TO}+\mathrm{O}_{\Gamma}$ transitions is observed. This small contribution is dominated by the low-energy tail at higher current values. The shape of the low-energy tail is weakly dependent on current or temperature, and can be described by an exponential function $\sim \exp \left(E / E_{0}\right)$ with $E_{0}$ energy of 42 $\mathrm{meV}$ and $13 \mathrm{meV}$ [Fig. 2(b)].

The exponential tails on the low-energy side suggest a possible explanation based on exponential localized tails of the conduction or valence bands. The exponential band tails are common for disordered materials, and the argument for disorder in $\mathrm{SiGe}$ is the existence of alloying fluctuations. By supposing exponential band tails, we have obtained relatively good fit for some of the EL spectra. However, the localized tail model disagrees qualitatively with the experimental data: The low-energy tails of the EL in this model must decrease with injection current. What we observe is that by increasing the current, the experimental curves in Fig. 2(a) show a slight increase of the low-energy tail relative to the spectral maximum. Therefore, we are forced to conclude that the low-energy tail of the EL spectra is caused by other phenomena. These can be Landsberg broadening or plasmon/ multiphonon replica.

In order to find out the appropriate fitting to the lowenergy tails, we performed for two current values, four fittings with different formulas for the broadening parameter and the carrier distribution function:

(1) Zero broadening parameter $\left[\Gamma_{h}=0, \rho_{h}^{\text {conv }}=\rho_{h}\right.$, Eq. (A2)], Figs. 4(a) and 4(b);

(2) Lorentzian convolution of the carrier distribution with Landsberg broadening function $\left[\Gamma_{h}\right.$ with Eq. (A8), $\rho_{h}^{\text {conv }}$ with Eq. (A5)], Figs. 4(c) and 4(d);

(3) Lorentzian convolution of the state distribution with Landsberg broadening function $\left[\Gamma_{h}\right.$ with Eq. (A8), $\rho_{h}^{\text {conv }}$ with Eq. (A10)], Figs. 4(e) and (f); and

(4) Constant broadening parameter $\left[\Gamma_{h}=\right.$ constant value, $\rho_{h}^{\text {conv }}=\rho_{h}^{\text {conv }}$ of Eq. (A10)], Figs. $4(\mathrm{~g})$ and (h).

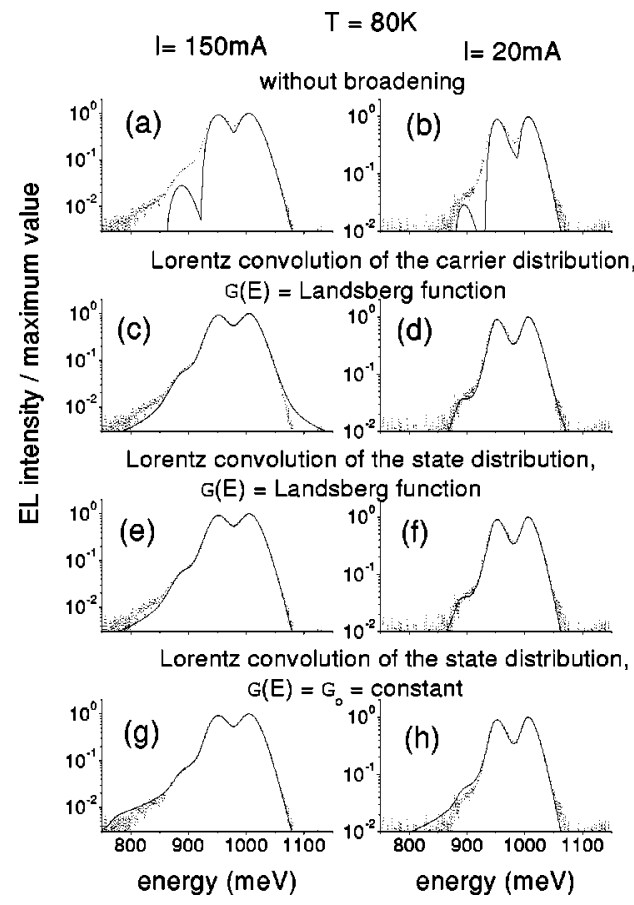

FIG. 4. Fitting curves (full lines) and experimental spectral EL (dotted lines) at $80 \mathrm{~K}$ for two current values $150 \mathrm{~mA}$ [(a), (c), (e), and (g)] and $20 \mathrm{~mA}$ [(b), (d), (f), and (h)]: (a), (b) No broadening; (c), (d) Lorentz convolution of carrier distributions with Landsberg function $\Gamma(E)$; (e), (f) Lorentz convolution of state distributions with Landsberg function $\Gamma(E)$; and $(\mathrm{g})$, (h) Lorentz convolution of state distributions with $\Gamma(E)=$ constant.

For all of the cases, the electron broadening parameter was approximated to a zero value $\left[\Gamma_{e}=0, \rho_{e}^{\text {conv }}=\rho_{e}\right.$ Eq. (A2) $]$ due to the smaller conduction band filling effect, as will be discussed next. The calculated spectra were obtained by a superposition of the NP maximum with phonon replicas $\mathrm{TO}_{\mathrm{Si}-\mathrm{Si}}$, transverse acoustic, $\mathrm{TO}_{\mathrm{Si}-\mathrm{Ge}}$, and $\mathrm{TO}+\mathrm{O}_{\Gamma}$ with the relative intensities $r_{i}$ of $80 \%-90 \%, 10 \%, 7 \%-15 \%, 2 \%-3 \%$, and phonon energies $E_{\text {phon }}$ of 55-56 meV, $16 \mathrm{meV}, 49 \mathrm{meV}$, and $112 \mathrm{meV}$, respectively.

Figure 4 shows that except for the zero broadening case, the low-energy tail can be reasonably fitted for all broadening cases. Thus, the Lorentzian convolution gives a good simulation of the logarithmic slope [Fig. 2(b)] with only one parameter $\Gamma$. However, the Landsberg procedure with the convolution applied to the carrier distribution gives a slow slope tail also for the high-energy side, in addition to the tail given by the Fermi occupation function. This is observed for the high current curve in Fig. 4(c). The additional Landsberg tail at the high-energy side of the spectrum appears in the theoretical curves at all injection values. In Fig. 4(d), the Landsberg high-energy tail appears at a lower EL intensity than the experimental measurement range. Besides this qualitative discrepancy, the fitting parameter $\Gamma_{0}$ in Eq. (A8) differs for the zero-temperature estimation of the Landsberg formula. ${ }^{31,14}$ For example, an estimation for the valence Fermi level of $12 \mathrm{meV}$ [at $20 \mathrm{~mA}$, Fig. 4(d)] gives a value of a $\Gamma_{0}=5.5 \mathrm{meV}$. From the fit, a $\Gamma_{0}$ value, nearly independent of the injection level, is obtained with a higher value [Figs. 4(c) and 4(d)] than the Landsberg estimation. At lower injection levels, the tail is reduced by the decrease of the Fermi 
level and the parameter $\Gamma_{0}$ remains almost constant. Similar results have been reported in Ref. 18.

The qualitative discrepancy can be removed by considering the effective carrier distribution given by Eq. (A10) where the Fermi function is outside the convolution integral [Figs. 2(e) and 2(f)]. This approximation corresponds to the Lorentzian convolution of the density of state distribution instead of a convolution of the carrier distribution function, and some physical reasons for this approximation were discussed in Sec. IV B.

This better fit of the experimental data with the Fermi function outside the convolution integral raises fundamental question: (1) Is the low-energy tail controlled by the finite lifetime of the final state of the EHP assembly or (2) by the finite lifetime of the unielectron states due to the multiple interaction in the EHP system? The aforementioned results suggest that for high injection levels, the broadening of the initial unielectronic states is more important for the lowenergy tail of the light emission.

For a constant $\Gamma$ value and a Lorentzian convolution applied to the density of states distribution [Eq. (A10) and $\Gamma=$ constant $]$, a good fit of the experimental curves is obtained [Figs. 4(h) and 4(g)]. In this case, for lower injection levels, the tails can be reduced by reducing the $\Gamma$ value. This approach has the advantage that the convolution in Eq. (A10) has an analytic form for parabolic bands.

We can see that depending on the fitting procedure the plasma parameters change slightly for the same experimental curve, regarding, for example, the concentration $p$ and the renormalized gap $E_{g}^{*}$ [Fig. 4]. The fitting results using the last procedure with a constant value for $\Gamma$, are shown in Figs. 5-7. To calculate the density of states distribution $D_{e, h}(\epsilon)$ $=n_{v}^{e, h} \sqrt{2 m_{\|}^{e, h}\left(m_{\perp}^{e, h}\right)^{2}} m_{0}^{3 / 2} / \pi^{2} \hbar^{3} \epsilon^{1 / 2}$, information on the band structure is needed, i.e., the equivalent valley number $n_{v}^{e, h}$ and the effective masses in $m_{0}$ units $m_{\|}^{e, h}, m_{\perp}^{e, h}$.

In $\mathrm{Si}_{1-x} \mathrm{Ge}_{x} / \mathrm{Si}$ epitaxial layers, the uniaxial stress changes the electronic band structure by splitting the degenerate bands. ${ }^{24}$ The heavy-hole band becomes the upper valence band. For $x=0.20$, the layers are coherently strained, thus, the splitting energy between the heavy and light hole bands can be estimated to $40 \mathrm{meV}^{33}$ In addition, the accumulation of holes in the upper valence band can increase the splitting of hole bands by affecting the mostly filled valley. ${ }^{34}$ The strain induces also splitting of the six equivalent $\Delta$ minima of the Si-type conduction band. The minimum of the conduction band in compressively strained SiGe consist of four equivalent valleys. The splitting energy is above 40 $\mathrm{meV}$ for $x=0.20$ and even higher than for the valence band. ${ }^{24}$ Therefore, we can consider for both bands the approximation of only one band model with equivalent valleys $n_{v}^{h}=1$ and $n_{v}^{e}=4$. The heavy hole effective masses in strained SiGe were theoretically estimated in Ref. 35 for $x$ $=0.20: m_{\|}^{h}=0.19$ and $m_{\perp}^{h}=0.27$. For the conduction band, the strain effect on the effective electron masses is negligible: $m_{\|}^{e}=0.916$ and $m_{\perp}^{e}=0.19 .{ }^{24}$ Due to the higher number of equivalent valleys and the higher effective mass product in the density of states expression, the quasi-Fermi level is lower in the conduction band. This relatively reduced con-
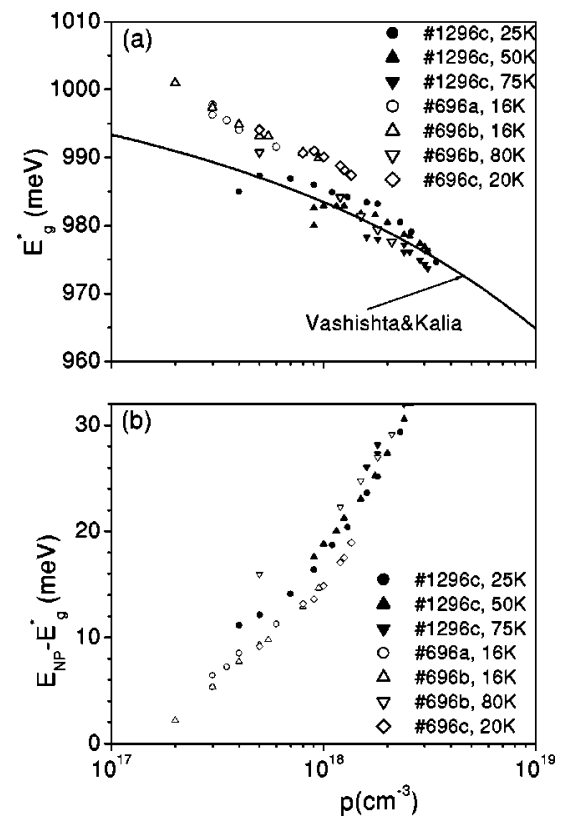

FIG. 5. Hole concentration dependencies of: (a) Renormalized band gap $E_{g}^{*}$ and (b) NP peak position relative to $E_{g}^{*}$. Points are fitting results for spectra on samples and temperatures mentioned in the graphs (sample notation as in Fig. 3). The theoretical curve in (a) was computed with the Vashishta and Kalia formula. The diode types: No. 1296c-100 diodes of area 24 $\times 24 \mu \mathrm{m}^{2}$; sample Nos. 696a, b, and c are diodes of an area of 1 $\times 1 \mathrm{~mm}^{2}, 0.5 \times 0.5 \mathrm{~mm}^{2}$, and $0.2 \times 0.2 \mathrm{~mm}^{2}$.

duction band filling justifies the approximation used here of negligible tails for the conduction band.

The renormalized energy gap is obtained by EHP-EL shape analysis for different samples at various temperatures and injection levels. The data are shown in Fig. 5(a) in comparison with the computed curve based on the Vashishta and Kalia formula, Eqs. (1) and (2). The values for the exciton
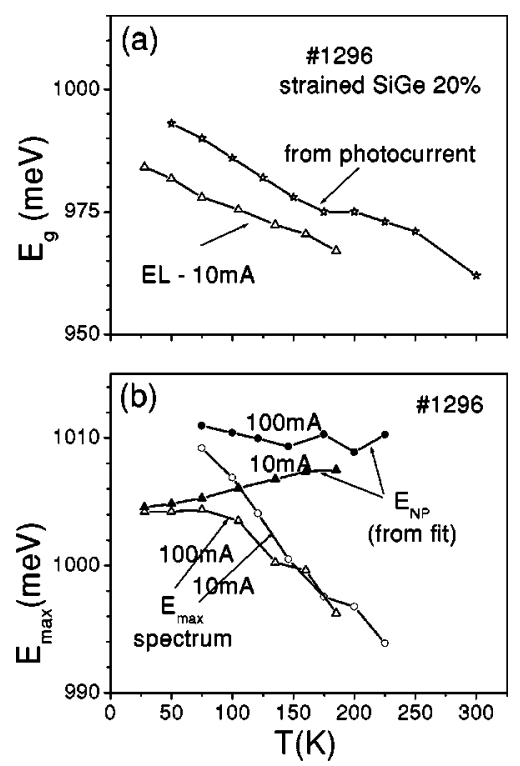

FIG. 6. Temperature dependence of: (a) Renormalized band gap in comparison with the optical band gap evaluated from the spectral photocurrent (see Ref. 8), and (b) The maximum energies of NP peak $E_{\mathrm{NP}}$ from fit and the experimental peak position of the total spectrum. The EHP band filling results are for $10 \mathrm{~mA}$ and $100 \mathrm{~mA}$. 


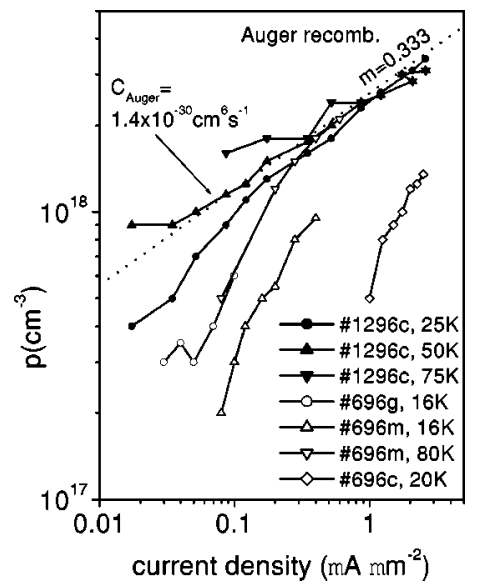

FIG. 7. Current dependence of the hole concentration obtained from the analysis based on EHP band filling model. Sample No. 1296 has $d_{\mathrm{SiGe}}$ $=260 \mathrm{~nm}$ and sample No. 696 has $d_{\mathrm{SiGe}}=60 \mathrm{~nm}$. The dotted line corresponds to Auger recombination with the fitting parameters: Area $=5.76$ $\times 10^{-4} \mathrm{~cm}^{-2} ; d_{\mathrm{SiGe}}=260 \mathrm{~nm} ;$ and $C_{\text {Auger }}=1.4 \times 10^{-30} \mathrm{~cm}^{6} \mathrm{~s}^{-1}$.

Bohr radius of $4.83 \mathrm{~nm}$ and for Rydberg energy of $9.3 \mathrm{meV}$ were taken from Ref. 36. The intrinsic gap value $E_{g}$ $=1007 \mathrm{meV}$ was estimated for $x=0.20$ using the analytical expression given in Ref. 36.

The measurement temperature was in the range of $16-80 \mathrm{~K}$. The local temperature was found by the line shape fit and differs by less than $20 \mathrm{~K}$ from the substrate temperature, with a linear dependence on the applied electric power. In Fig. 5(a), the fitting data obtained from the EL spectra of sample No. 1296c are shown with full symbols, while the open symbols are for several diodes of epitaxy No. 696 (diodes with different areas). The theoretical computation and the points found by the shape analysis show a similar dependence of the renormalized gap on the plasma density. The experimental points are close to the computed curve, but centered in the upper side. A better knowledge of the intrinsic material parameters, or a better procedure to simulate the tails may give a better agreement with the Vashishta and Kalia formula. The band-gap narrowing due to doping also affects the results. Theoretical analysis of the $p$-type doping in $\mathrm{SiGe}$ gives a band-gap reduction above $50 \mathrm{meV}$ for $x$ $=0.20$ and $p=10^{18} \mathrm{~cm}^{-3}$ (Ref. 37). The data in Fig. 5(a) show a lower renormalized gap for sample No. 1296 with a $n$ doping of about $10^{18} \mathrm{~cm}^{-3}$, comparatively to sample No. 696 which has a lower $n$-type doping of $10^{16} \mathrm{~cm}^{-3}$ (see Table I).

Figure 5(b) shows the difference between the NP peak energy and the renormalized gap $E_{\mathrm{NP}}-E_{g}^{*}$. This difference increases at high injection levels, but, experimentally, only a small shift of the NP peak position is observed [Fig. 3] due to the compensation of the band filling by the reduction of the gap due to renormalization.

The renormalized gap decreases also by increasing the temperature as seen in Fig. 5(a) (experimental points are at $20 \mathrm{~K}$ and $75 \mathrm{~K}$ for No. 1296). The temperature dependence of the renormalized gap was found by extending the shape analysis at higher temperatures. In Fig. 6(a), the temperature dependence of the renormalized gap at low injection current is shown comparatively with the optical band gap obtained by spectral photocurrent measurements. ${ }^{8}$ The expected decrease with temperature is indeed observed. The small difference observed between the renormalized gap $E_{g}^{*}$ of EL analyzes and the optical gap $E_{g}$ obtained from photocurrent investigations can be explained by plasma effects which exist under EL conditions even at a low injection current, in contrast with very low injection conditions of the spectral photocurrent measurements.

The $E_{\mathrm{NP}}$ increases relative to the renormalized energy gap due to the thermal broadening of the distribution of injected carriers. This increase of $E_{\mathrm{NP}}$ may compensate for or exceed the temperature decrease of the band gap. In Fig. 6(b), $E_{\mathrm{NP}}$ obtained from the fit has a slow dependence on temperature for $100 \mathrm{~mA}$ and shows a blueshift for smaller injection current $10 \mathrm{~mA}$. However, due to the superposition of the NP and TO maxima, the apparent experimental NP peak has a value $E_{\max }$ (spectrum) which shows a fast redshift with temperature. Therefore, for obtaining reliable data about the temperature dependence of the NP peak or the band gap, careful spectral-shape analysis must be done.

The dependence of the plasma concentration on current density can be used to find the dominant recombination process. In Fig. 7, the $p(J)$ data for the samples and temperatures of Fig. 5 are shown. Sample No. 1296 shows a clear dominant Auger recombination with a dependence $p \sim J^{1 / 3}$. The straight line in Fig. 7 fits the EHP data well and corresponds to the total Auger coefficient $C_{\text {Auger }}=C_{e}+C_{h}=1.4$ $\times 10^{-30} \mathrm{~cm}^{6} \mathrm{~s}^{-1}$, close to the value found by the modeling of the current dependence of the quantum efficiency ${ }^{8}$ and to the values given in literature for $\mathrm{Si}$ and $\mathrm{Ge} .^{38}$

For our experiments, within the temperature range of $16-80 \mathrm{~K}$, the plasma density was found for most of the measurements above $2 \times 10^{17} \mathrm{~cm}^{-3}$. In this experimental range, the line shape of EL changes with the injection level as expected for an uniform spatial EHP distribution. Only under certain experimental conditions, such as low temperature $(16 \mathrm{~K})$ and low current density (large diode area and small injection current, $J<0.03 \mu \mathrm{A} / \mu \mathrm{m}^{2}$ ), the shape remains unchanged when the current changes. For these curves, the fitting procedure gives a constant density of $2-3 \times 10^{17} \mathrm{~cm}^{-3}$. This phenomena may be related to the alloy or/and doping fluctuations.

In conclusion, the shape analysis of the EL of strained SiGe has shown the dominance of EHP in the investigated injection level and temperature ranges. Good agreement with the theoretical predictions was found.

\section{CONCLUSIONS}

The line shape analysis of EL spectra was used to study the EHP in thick strained SiGe with $x=0.20$. In the case of thick layers, less influence on the EL spectra due to the interface $\mathrm{SiGe} / \mathrm{Si}$ regions is expected. The thickness of strained SiGe layer was increased up to ten times the critical thickness for plastic relaxation by using selective epitaxial growth of small square mesas of 20-200 $\mu \mathrm{m}$ width.

For the shape analysis, a superposition of NP and phonon replicas was considered. The spectra including the low- 
and high-energy tails are well described with the band filling model by the convolution of density of states distribution with a Lorentz function. Different procedures to describe the tails, including Landsberg broadening, were analyzed. The renormalized band gap in function of carrier concentration was obtained for different samples and compared with the theoretical prediction. The fitting points are close to the theoretical curve obtained using the universal formula of Vashishta and Kalia. The temperature dependence of the renormalized gap at low injection current and Auger recombination constant obtained by line shape analysis agree well with results obtained by spectral photocurrent measurements performed on the same diodes.

\section{ACKNOWLEDGMENTS}

The authors are grateful to Bernd Holländer for the RBS studies, Andreas Mück for the SIMS studies, and to Hilde Siekmann and Gunnar Schöpe for the $\mathrm{ZnO}$ deposition.

\section{APPENDIX: SPECTRAL FUNCTION OF LIGHT EMISSION IN ELECTRON-HOLE PLASMAS}

The spectral dependence of the NP emission can be written as:

$$
\begin{aligned}
\Psi_{\mathrm{EL}}^{\mathrm{NP}}\left(h \nu, E_{g}^{*}\right) \sim & \int_{0}^{\infty} \int_{0}^{\infty} d \epsilon_{e} d \epsilon_{h} M^{2} \rho_{e}\left(\epsilon_{e}\right) \rho_{h}\left(\epsilon_{h}\right) \delta\left(\epsilon_{e}+\epsilon_{h}\right. \\
& \left.+E_{g}^{*}-h \nu\right),
\end{aligned}
$$

with the carrier distribution functions:

$$
\rho_{e, h}(\epsilon)=D_{e, h}(\epsilon) f\left(\epsilon-\epsilon_{F}^{e, h}\right),
$$

where $D_{e}\left(\epsilon_{e}\right)$ and $D_{h}\left(\epsilon_{h}\right)$ are the density of states for conduction and valence bands; $f\left(\epsilon-\epsilon_{F}\right)=(1+\exp ((\epsilon$ $\left.\left.\left.-\epsilon_{F}\right)\right) / k T\right)^{-1}$ is the Fermi function for energy $\epsilon$ and quasiFermi level $\epsilon_{F} ; E_{g}^{*}$ is the renormalized gap; the $\delta$ function assures the energy conservation law. All energies are relative to the bottom of the corresponding band. The matrix element $\mathrm{M}$ of the transition probability involving the initial state in the conduction band and the final state in the valence band is considered to be weakly dependent on the energies of the initial and final states and is taken outside of the integral in Eq. (A1). The broadening of the maxima due to alloy and thermal fluctuations is neglected, parabolic bands $D_{e}(\epsilon)$, $D_{h}(\epsilon) \sim \epsilon^{1 / 2}$ are supposed in Eq. (A1).

For the light emission with phonon participation, the renormalized gap $E_{g}^{*}$ in Eq. (A1) must be replaced by $E_{g}^{*}$ $-E_{\text {phonon }}^{i}$, where $E_{\text {phonon }}^{i}$ is the energy of the emitted phonons in $i$-type phonon replica. The total emission is given by the superposition of NP and all phonon replicas:

$$
\Psi_{\mathrm{EL}}^{\mathrm{total}}(h \nu)=\sum_{i} r_{i} \Psi_{\mathrm{EL}}^{\mathrm{NP}}\left(h \nu, E_{g}^{*}-E_{\text {phonon }}^{i}\right),
$$

with $r_{i}$ the relative intensity phonon/NP emission.

The spatial neutrality condition must be also satisfied in an uniform EHP system:

$$
\int_{0}^{\infty} d \epsilon\left[D_{e}(\epsilon) f\left(\epsilon-\epsilon_{F}^{e}\right)-D_{h}(\epsilon) f\left(\epsilon-\epsilon_{F}^{h}\right)\right]=N_{d}-N_{a},
$$

with $N_{d}$ and $N_{a}$ the donor and acceptor concentrations.

In the case of the broadening of the final state of the electron-hole recombination process in EHP (Landsberg theory), the electron and hole carrier distribution functions $\rho_{e, h}(\epsilon)$ must be replaced by the convolutions with a broadening function $L(\epsilon, \Gamma)$.

$$
\begin{aligned}
\rho_{e, h}^{\text {conv }}(\epsilon)= & \int_{0}^{\infty} d \epsilon^{\prime} D_{e, h}\left(\epsilon^{\prime}\right) f\left(\epsilon^{\prime}-\epsilon_{F}^{e, h}\right) \\
& \times L\left(\epsilon^{\prime}-\epsilon, \Gamma_{e, h}\left(\epsilon^{\prime}\right)\right),
\end{aligned}
$$

where $\Gamma$ is the broadening parameter. Equation (A1) becomes

$$
\begin{aligned}
\Psi_{\mathrm{EL}}^{\mathrm{NP}}\left(h \nu, E_{g}^{*}\right) \sim & \int_{0}^{\infty} \int_{0}^{\infty} d \epsilon_{e} d \epsilon_{h} \rho_{e}^{\mathrm{conv}}\left(\epsilon_{e}\right) \rho_{h}^{\mathrm{conv}}\left(\epsilon_{h}\right) \\
& \times \delta\left(\epsilon_{e}+\epsilon_{h}+E_{g}^{*}-h \nu\right) .
\end{aligned}
$$

For broadening of the electronic states due to the energy uncertainty, the Lorentz distribution function:

$$
L\left(\epsilon^{\prime}-\epsilon, \Gamma_{e, h}\left(\epsilon^{\prime}\right)\right)=\frac{1}{\pi} \frac{\Gamma_{e, h}\left(\epsilon^{\prime}\right)}{\left(\epsilon^{\prime}-\epsilon\right)^{2}+\left[\Gamma_{e, h}\left(\epsilon^{\prime}\right)\right]^{2}},
$$

can be used in Eq. (A5). The broadening parameter $\Gamma_{e, h}(\epsilon)$ obtained for carrier relaxation at $0 \mathrm{~K}$ by Auger effect ${ }^{31}$ has a complicated form. A simplified polynomial one is usually taken. ${ }^{14}$ This can be written in the equivalent form:

$$
\Gamma_{e, h}(\epsilon)=\Gamma_{0}^{e, h}\left[1-\frac{\epsilon}{\epsilon_{F}^{e, h}}\right]^{2}\left[1-0.229 \frac{\epsilon}{\epsilon_{F}^{e, h}}\right] .
$$

Using Fourier transform procedures, the spectral dependence in Eq. (A6) can be transformed into:

$$
\begin{aligned}
\Psi_{\mathrm{EL}}^{\mathrm{NP}}\left(h \nu, E_{g}^{*}\right) \sim & \int_{0}^{\infty} \int_{0}^{\infty} d \epsilon_{e} d \epsilon_{h} \rho_{e}\left(\epsilon_{e}\right) \rho_{h}\left(\epsilon_{h}\right) L\left(\epsilon_{e}+\epsilon_{h}\right. \\
& \left.+E_{g}^{*}-h \nu, \Gamma\left(\epsilon_{e}, \epsilon_{h}\right)\right),
\end{aligned}
$$

where $\Gamma\left(\epsilon_{e}, \epsilon_{h}\right)=\Gamma_{e}\left(\epsilon_{e}\right)+\Gamma_{h}\left(\epsilon_{h}\right)$ is the sum of broadening parameters of the conduction and valence bands given by Eq. (A8). Equation (A9) shows that the Landsberg broadening corresponds also to the exchange of the $\delta$ function in Eq. (A1) by a Lorentz function.

In the case of the initial state broadening, the Fermi function appears outside the integral in Eq. (A5) and the equivalent carrier distribution is

$$
\begin{aligned}
\rho_{e, h}^{\mathrm{conv}}(\epsilon)= & f\left(\epsilon-\epsilon_{F}^{e, h}\right) \int_{0}^{\infty} d \epsilon^{\prime} D_{e, h}\left(\epsilon^{\prime}\right) \\
& \times L\left(\epsilon^{\prime}-\epsilon, \Gamma_{e, h}\left(\epsilon^{\prime}\right)\right) .
\end{aligned}
$$

For the simplified case of the constant $\Gamma$ value, the convolution of the Lorentz function with the density of states distribution [Eq. (A10)] has an analytic form for parabolic bands $D_{e, h} \sim \epsilon^{1 / 2}$ : 


$$
\begin{aligned}
& \int_{0}^{\infty} d \epsilon^{\prime} D_{e, h}\left(\epsilon^{\prime}\right) L\left(\epsilon^{\prime}-\epsilon, \Gamma\right) \\
& \sim \frac{\sqrt{2}}{\pi} \frac{\Gamma}{\sqrt{\epsilon^{*}-\epsilon}} \arctan \sqrt{\frac{\epsilon^{*}+\epsilon}{\epsilon^{*}-\epsilon}}
\end{aligned}
$$

with $\epsilon^{*}=\sqrt{\epsilon^{2}+\Gamma^{2}}$.

${ }^{1}$ G. Masini, L. Colace, and G. Assanto, Mater. Sci. Eng., B 89, 2 (2002).

${ }^{2}$ D. Dutartre, G. Bremound, A. Souifi, and T. Benyattou, Phys. Rev. B 44, 11525 (1991).

${ }^{3}$ X. Xiao, C. W. Liu, J. C. Sturm, and L. C. Lenchyshyn, and M. L. W. Thewalt, Appl. Phys. Lett. 60, 1720 (1992).

${ }^{4}$ L. Vescan, K. Schmidt, C. Dieker, H. P. Tang, T. Vescan, and H. Lüth, Thin Solid Films 222, 5 (1992).

${ }^{5}$ T. Stoica and L. Vescan, Semicond. Sci. Technol. 18, 409 (2003).

${ }^{6}$ L. Vescan and T. Stoica, J. Lumin. 80, 485 (1999).

${ }^{7}$ T. Stoica and L. Vescan, J. Cryst. Growth 131, 32 (1993).

${ }^{8}$ T. Stoica and L. Vescan, J. Appl. Phys. 93, 4461 (2003).

${ }^{9}$ T. Stoica, L. Vescan, and M. Goryll, J. Appl. Phys. 83, 3367 (1998).

${ }^{10}$ L. Vescan and T. Stoica, Proc. SPIE 3630, 163 (1999).

${ }^{11}$ G. B. Norris and K. K. Bajaj, Phys. Rev. B 26, 6706 (1982).

${ }^{12}$ I. Balslev, Phys. Rev. B 30, 3203 (1984).

${ }^{13}$ C. F. Klingshirn, Semiconductor Optics (Springer, Berlin, 1997), p. 306

${ }^{14}$ R. W. Martin and H. L. Störmer, Solid State Commun. 22, 523 (1977).

${ }^{15}$ W. Schmid, Phys. Status Solidi B 94, 413 (1979).

${ }^{16}$ A. Forchel, B. Laurich, J. Wagner, W. Schmid, and T. L. Reinecke, Phys. Rev. B 25, 2730 (1982).

${ }^{17}$ A. H. Simon, S. J. Kirch, and J. P. Wolfe, Phys. Rev. B 46, 10098 (1992).

${ }^{18}$ L. M. Smith and J. P. Wolfe, Phys. Rev. B 51, 7521 (1995).

${ }^{19}$ M. Combescot, O. B. Matibet, and C. B. Guillaume, Phys. Rev. B 53, 3861 (1996).
${ }^{20}$ K. H. Wang, M. Bayer, P. Ils, A. Forchel, S. Benner, H. Haug, P. P. Rossiaux, and L. Goldstein, Solid-State Electron. 40, 287 (1996).

${ }^{21}$ L. Vescan, C. Dieker, A. Hartmann, and A. van der Hart, Semicond. Sci. Technol. 9, 387 (1994).

${ }^{22}$ L. Vescan, T. Stoica, O. Chretien, M. Goryll, E. Mateeva, and A. Mück, J. Appl. Phys. 87, 7275 (2000).

${ }^{23} \mathrm{R}$. Apetz and L. Vescan (unpublished).

${ }^{24}$ Silicon Germanium and SiGe:Carbon, EMIS Data Reviews Series No. 24, edited by E. Kasper and K. Lyutovich, (INSPEC, London, 2000).

${ }^{25}$ T. M. Rice, Solid State Physics, edited by H. Ehrenreich, F. Setz, and D. Turnbull (Academic, New York, 1977), Vol. 32.

${ }^{26}$ P. Vashishta and R. K. Kalia, Phys. Rev. B 25, 6492 (1982).

${ }^{27}$ L. Banyai and S. W. Koch, Z. Phys. B: Condens. Matter 63, 283 (1986).

${ }^{28}$ R. C. C. Leite, J. C. Sarace, D. H. Olson, B. G. Cohen, J. M. Whelan, and A. Yariv, Phys. Rev. 137, A1583 (1965).

${ }^{29}$ E. Cohen, M. D. Sturge, M. A. Olmstead, and R. A. Logan, Phys. Rev. B 22, 771 (1980).

${ }^{30}$ M. Capizzi, S. Modesti, A. Frova, J. L. Staeli, M. Guzzi, and R. A. Logan, Phys. Rev. B 29, 2028 (1984).

${ }^{31}$ P. T. Landsberg, Phys. Status Solidi 15, 623 (1966).

${ }^{32}$ C. H. Aldrich and R. N. Silver, Phys. Rev. B 21, 600 (1980).

${ }^{33}$ C. G. Van de Walle, Phys. Rev. B 39, 1871 (1989).

${ }^{34}$ H. Kalt and M. Rinker, Phys. Rev. B 45, 1139 (1992).

${ }^{35}$ R. Loo, L. Vescan, A. Hartmann, R. Apetz, U. Zastrow, T. Schäpers, A. Leuther, C. Dieker, H. Lüth, P. Gartner, and T. Stoica, Phys. Rev. B 50, 18113 (1994).

${ }^{36}$ D. J. Robbins, L. T. Canham, A. D. Pitt, and P. Calcott, J. Appl. Phys. 71, 1407 (1992).

${ }^{37}$ J. Poortmans, S. C. Jain, D. H. J. Totterdell, M. Caymax, J. F. Nus, R. P. Mertens, and R. Van Overstraeten, Solid-State Electron. 35, 1763 (1993).

${ }^{38}$ Handbook Series on Semiconductor Parameters, Vol. 1, edited by M. Levinshtein, S. Rumyantsev, M. Shur, and Ya. Pokrovskii (World Scientific, Singapore, 1996), Chap. 1. 